\title{
The impact of sarcopenia on health-related quality of life in elderly people: Korean National Health and Nutrition Examination Survey
}

\author{
Der Sheng Sun ${ }^{1}$, Hyunyong Lee ${ }^{2}$, Hyeon Woo Yim ${ }^{3}$, Hye Sung Won ${ }^{1}$, and Yoon Ho Ko ${ }^{1}$
}

\begin{abstract}
${ }^{1}$ Department of Internal Medicine, College of Medicine, Uijeongbu St. Mary's Hospital, The Catholic University of Korea, Uijeongbu; ${ }^{2}$ Clinical Research Coordinating Center, ${ }^{3}$ Department of Preventive Medicine, College of Medicine, The Catholic University of Korea, Seoul, Korea
\end{abstract}

Received: May 18, 2017

Revised : July 11, 2017

Accepted: August 4, 2017

\section{Correspondence to}

Hye Sung Won, M.D.

Department of Internal Medicine, College of Medicine,

Uijeongbu St. Mary's Hospital, The Catholic University of Korea, 271 Cheonbo-ro, Uijeongbu 11765, Korea

Tel: +82-31-820-5247

Fax: +82-31-847-2719

E-mail: woncomet@catholic.ac.kr

Yoon Ho Ko, M.D.

Department of Internal Medicine, College of Medicine, Uijeongbu

St. Mary's Hospital, The Catholic University of Korea, 271 Cheonbo-ro, Uijeongbu 11765, Korea

Tel: $+82-31-820-3985$

Fax: +82-31-847-2719

E-mail: koyoonho@catholic.ac.kr
Background/Aims: The purpose of the study is to investigate the associations between sarcopenia and health-related quality of life in elderly men and women in Korea.

Methods: In a cross-sectional study using data from 2008 to 2011 Korean National Health and Nutrition Examination Survey, 4,937 adults aged 6o years and older who underwent a dual-energy X-ray absorptiometry scan were included in the study. Sarcopenia is defined as an appendicular skeletal muscle index of two standard deviations or more below the mean for young, healthy reference populations. The health-related quality of life was measured using the EuroQol-5 dimension questionnaire.

Results: The overall prevalence of sarcopenia was $6.6 \%$ in these Korean people over the age of 60 years: $11.1 \%$ for men and $3.2 \%$ for women. Sarcopenic men tended to have lower income, lower physical activity, lower body mass index, and smaller waist circumference compared with nonsarcopenic men. Sarcopenic women tended to have higher body mass index and larger waist circumference compared with nonsarcopenic women. Sarcopenic men showed higher impairments in mobility, self-care, usual activities, and pain/discomfort compared with nonsarcopenic men. Women with sarcopenia also showed higher impairments in mobility, self-care, usual activities, and anxiety/depression compared with nonsarcopenic women. Sarcopenia showed an association with impairments in selfcare for men, and with impairments in self-care, usual activities, and anxiety/ depression for women, after adjusting for other confounding factors.

Conclusions: There is a significant association between sarcopenia and impaired health-related quality of life in this elderly Korean population, and these results differ between men and women.

Keywords: Sarcopenia; Aged; Quality of life

\section{INTRODUCTION}

The world is rapidly moving into an aged society. As life expectancy is extended, the health and socioeconomic burden of ageing-related problems are increasing expo- nentially. In recent years, the interest in the quality of life (QOL) of elderly people has also increased. Many experts and older people believe that the QOL is more important than length of life. QOL is a broad multidimensional concept. The World Health Organization defines 
the concept of QOL as 'individual's perception of their position in life in the context of the culture and value systems in which they live and in relation to their goals, expectations, standard and concerns' [1]. The elderly are generally vulnerable due to declining physical and mental capabilities, and therefore health-related quality of life (HRQOL) may be most important issue. As a result, it is challenging to improve their life by investigating factors related to HRQOL in the elderly.

There have been many advances in knowledge about aging processes. Aging is accompanied by many changes in body composition, and one of these is sarcopenia [2]. Sarcopenia is defined as a decrease of skeletal muscle mass and function, and has emerged as one of the most common problems in the elderly population [3]. The estimated prevalence of sarcopenia ranges from 5\% to $13 \%$ among people aged 60 years and over, increasing to as high as 50\% in people over the age of 80 years [4].

Originally, Baumgartner et al. [5] defined sarcopenia as being two standard deviations below the normal appendicular skeletal muscle mass divided by height squared. Dual-energy X-ray absorptiometry (DXA), an accurate and reliable method to distinguish fat, bone, and lean body mass, is the most widely used method for measuring muscle mass in sarcopenia research [6].

In recent years, there has been a strong interest in the study of sarcopenia, and evidence for its significance and impacts in the elderly has been growing [6]. Sarcopenia can have detrimental effects on physical and functional activities and on the quality of life in elderly groups [7]. However, most studies about the relationship between sarcopenia and QOL have been conducted in Western populations, and few studies have been undertaken among the elderly in Korea. Therefore, here we aimed to investigate the impact of sarcopenia on QOL among elderly Koreans using nationally representative data.

\section{METHODS}

\section{Study population}

We used data obtained from the Korean National Health and Nutrition Examination Survey (KNHANES IV to V) from 2008 to 2011 . This was a cross-sectional study conducted every year by the Korea Centers for Disease Control and Prevention. The sampling units were registered households selected through a stratified, multistage probability-sampling design considering geographic area, gender, and age, based on data from the Population and Housing Census and the Korean registered population. Therefore, the sample was representative of the noninstitutionalized civilian Korean population. Among 37,753 individuals who participated in the survey, 4,937 adults aged 60 years and older who underwent a DXA scan were included. This survey was approved by the Institutional Review Board of Korea Centers for Disease Control and Prevention (IRB: 2008-04EXP-o1-C, 2009-01CON-03-2C, 2010-02CON-21-C, 2011-02CONo6-C). And all the participants in this survey signed an informed consent form. The data are available on the website (http://knhanes.cdc.go.kr/) [8].

\section{Data collection}

The KNHANES consisted of three components: a health survey, a nutritional intake survey, and a health examination. The health survey included information on smoking, alcohol consumption, physical activity, mental health, comorbidity, educational and economic status, and HRQOL. Current smokers were defined as those who had smoked greater than 100 cigarettes in their life and who currently smoked cigarettes. Heavy alcohol drinking was defined as consuming seven or more standard drinks a week for men, and five or more standard drinks a week for women. Physical activity levels were divided into three categories: (1) high, vigorous activities that make the subject breathe hard for 20 minutes at least 3 days a week; (2) moderate, activities that make the subject breathe a little harder for 30 minutes at least 5 days a week; and (3) low, common daily activities that do not require much effort.

The HRQOL was measured using the EuroQol-5 dimension (EQ-5D), which is the most commonly used standardized questionnaire for measuring health outcomes $[9,10]$. The EQ-5D consists of the EQ-5D descriptive system and the EQ visual analogue scale (EQ-VAS). The EQ-5D descriptive system comprises five dimensions: mobility, self-care, usual activities, anxiety/depression, and pain/discomfort. Each dimension has three levels: no problems, some problems, and extreme problems. The EQ-5D descriptive system can be converted into a weighted health state index score by applying scores from the EQ-5D preference weights elicited from 
general population samples. The EQ-5D index score ranges from states worse than dead $(-1)$ to full health (1), anchoring death at $\mathrm{o}$. For this study, Korean population weightings were used to convert the results to an EQ${ }_{5} \mathrm{D}$ index score. The EQ-VAS records the respondent's self-reported health on a vertical, VAS from o (worst imaginable health) to 100 (best imaginable health).

Health examinations included anthropometric measurements, blood and urine tests, chest X-rays, ophthalmic and oral examinations, and bone mineral density measurements using DXA scans. All physical examinations were performed by a specially trained examiner who followed a standard procedure. Waist circumference was measured in the horizontal plane midway between the iliac crest and costal margin at the end of a normal expiration. Body mass index (BMI) was calculated as body weight in kilograms divided by the square of height in meters.

\section{Study variables}

We measured the total body fat tissue and lean soft tissues in kilograms using DXA scans. Lean soft tissue mass was separated into trunk and appendicular components, and appendicular lean mass was calculated as the sum of lean soft tissue mass in the arms and legs. The skeletal muscle index (SMI) was obtained by dividing absolute muscle mass by squared height $\left(\mathrm{kg} / \mathrm{m}^{2}\right)$. According to Baumgartner's criteria, we defined sarcopenia as an appendicular SMI of two standard deviations or more below the mean for young, healthy reference populations [5]. According to this definition, the cut-off values were $6.03 \mathrm{~kg} / \mathrm{m}^{2}$ in men and $4.14 \mathrm{~kg} / \mathrm{m}^{2}$ in women.

Total body fat percentage was calculated as the weight of total body fat tissue divided by total body weight. Women with > 38\% total body fat and men with > 27\% total body fat were classified as obese [11].

To investigate the relationship between sarcopenia and HRQOL, we used the EQ-5D questionnaire including the EQ-5D descriptive system, the EQ-VAS, and the EQ-5D index score. The EQ-VAS and EQ-5D index score are presented as the mean \pm standard error and some or extreme problems reported in the EQ-5D descriptive system were categorized as indicating an impaired HRQOL.

\section{Statistical analyses}

Data are expressed as percentages or as the mean \pm stan- dard error. Student $t$ tests for continuous variables and chi-square tests for categorical variables were used to evaluate significant differences. Multivariable adjusted logistic analysis was performed to calculate odds ratios and $95 \%$ confidence intervals for the association of sarcopenia with HRQOL. All statistical analyses were performed using SAS version 9.4 (SAS Institute Inc., Cary, NC, USA). A $p$ value of less than 0.05 was considered to indicate statistical significance.

\section{RESULTS}

\section{Prevalence of sarcopenia}

A flow diagram for the study population is shown in Fig. 1. Among 4,937 adults over the age of 60 years, 324 had sarcopenia: an overall prevalence of $6.6 \%$. In terms of age groups, the prevalence of sarcopenia was $4.6 \%$, $7.3 \%$, and $15.7 \%$ in adults aged 60 to 69,70 to 79 , and $\geq 80$ years, respectively. The overall prevalence of sarcopenia was $11.1 \%$ for men and $3.2 \%$ for women, so men had a higher prevalence of sarcopenia than did women.

\section{Baseline characteristics associated with sarcopenia}

Table 1 summarized the characteristics of these elderly men and women according to the presence of sarcopenia. The mean age was higher in men and women with sarcopenia than in those without sarcopenia. Sarcopenic men tended to have a lower income, lower

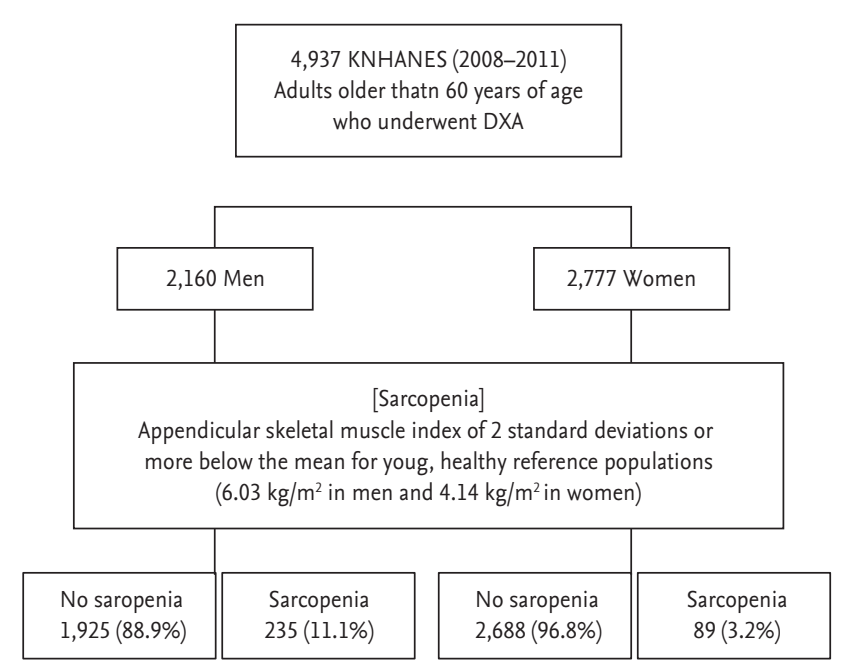

Figure 1. Flow diagram for the study population. KNHANES, Korean National Health and Nutrition Examination Survey; DXA, dual-energy X-ray absorptiometry. 


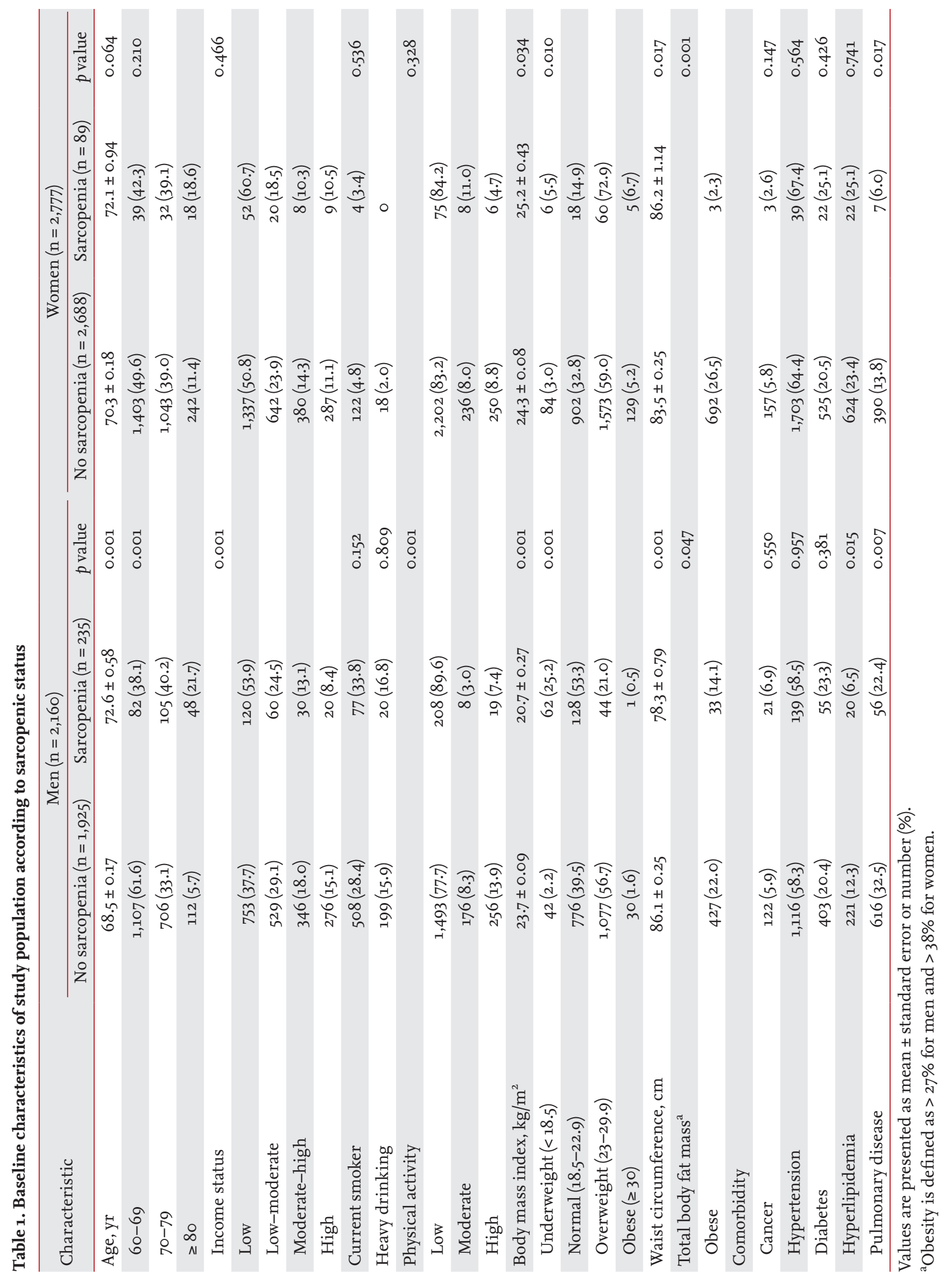




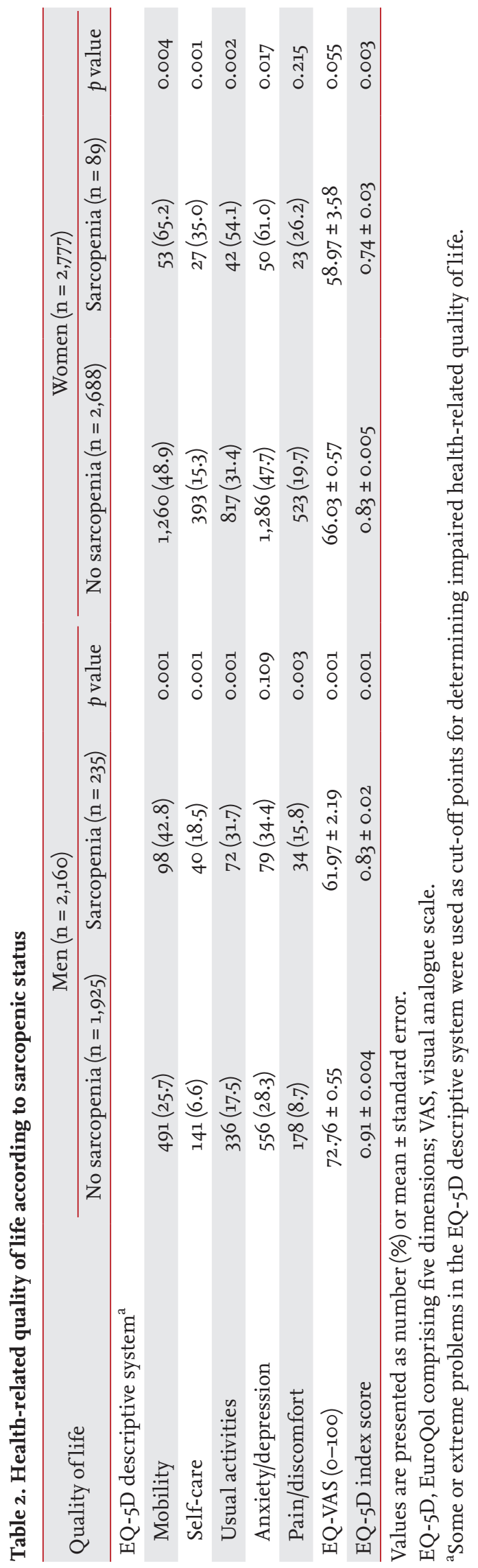

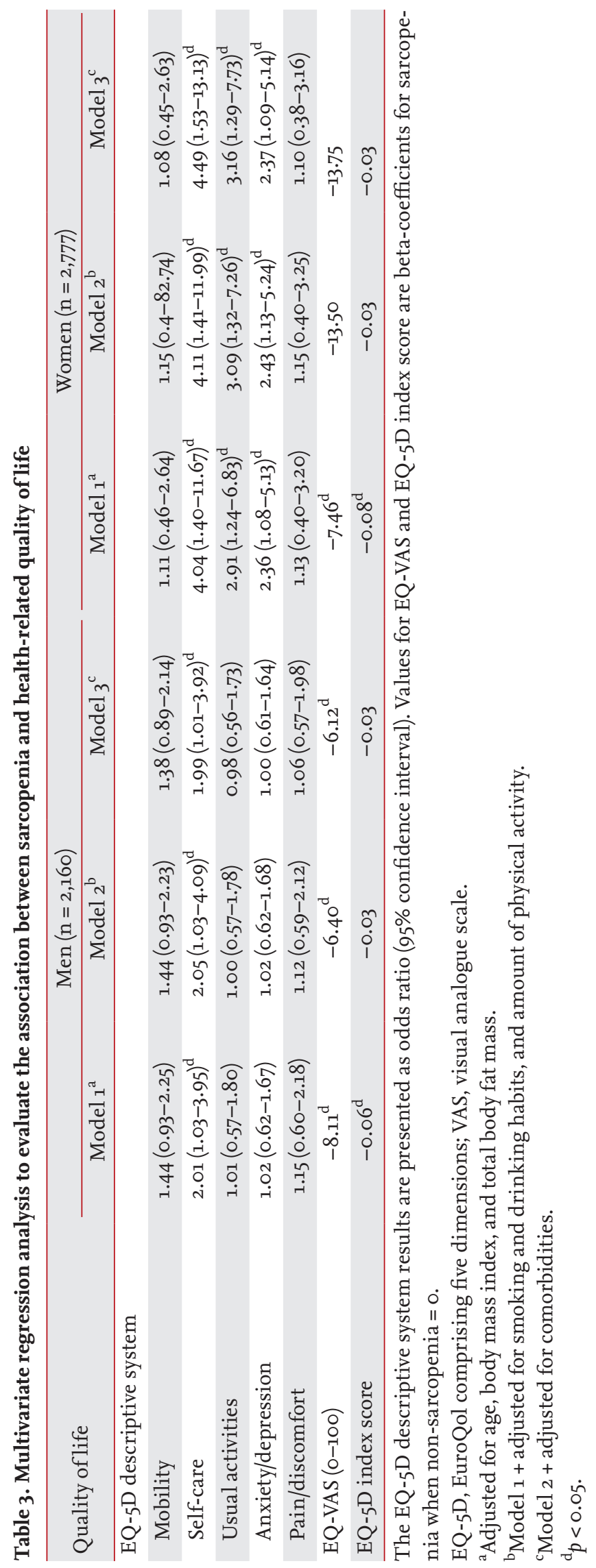


physical activity, lower BMI, and smaller waist circumference than men without sarcopenia. On the other hand, sarcopenic women tended to have a higher BMI and greater waist circumference than women without sarcopenia. In other words, sarcopenic obesity was more common in elderly women than in men. The prevalence of sarcopenia was lower among obese men and women based on total body fat percentages compared with nonobese men and women. There were no differences in smoking status, heavy drinking rates, comorbidities except pulmonary disease between sarcopenic and nonsarcopenic subjects.

\section{Associations between sarcopenia and health-related quality of life}

Table 2 showed the HRQOL using EQ-5D according to the presence of sarcopenia. In this descriptive system, sarcopenic men showed higher impairments in mobility, self-care, and usual activities, and more pain/ discomfort compared with nonsarcopenic men. Sarcopenic women also showed higher impairments in mobility, self-care, and usual activities, and more anxiety/ depression compared with nonsarcopenic women. The mean EQ-VAS and EQ-5D index scores were significantly lower for sarcopenic men and women compared with nonsarcopenic subjects.

Table 3 showed the results of a multivariate regression model used to evaluate the impact of sarcopenia on HRQOL. We determined three categories as factors that could affect the HRQOL: (1) age and obesity; (2) lifestyle; and (3) comorbidities. We analyzed the association between sarcopenia and HRQOL after adjusting for each factor. After adjusting for age, BMI, and total body fat mass, there was a significant association between sarcopenia and worse EQ-VAS and EQ-5D index scores in these elderly men and women. Within the EQ-5D descriptive system, impairments in self-care for men and in self-care and usual activities, and more anxiety/ depression for women were associated with sarcopenia. Sarcopenia showed an association with worse EQ-VAS, impairments in self-care for men and in self-care and usual activities, and more anxiety/depression for women, even after adjusting for all three categories. The adjusted odds ratios for self-care were 1.99 and 4.49 for sarcopenic men and women, respectively.

\section{DISCUSSION}

Our study showed that $11.1 \%$ for men and 3.2\% for women over 60 years of age were sarcopenic, and sarcopenia had a significant association with the HRQOL in Korean elderly people.

The prevalence of sarcopenia has been reported at about $5 \%$ to $10 \%$ in those over 60 years of age, but it varies according to age, ethnicity, definition of sarcopenia, and disease status. Globally, the prevalence of sarcopenia has been reported to be higher in men. Kim et al. [12] reported that the prevalence of sarcopenia in the elderly Korean population in 2012 was $12.4 \%$ for men and $0.1 \%$ for women.

The pathogenesis of sarcopenia is considered multifactorial: decreased physical activity; age-related mitochondrial dysfunction; loss of motor neuron end plates; weight loss; loss of anabolic hormones; and increases in proinflammatory cytokines have been implicated [6]. Sarcopenia caused by these various degenerative facts leads to a negative impact on general health in elderly populations. Sarcopenia is known to be associated with poor physical performance and functional decline. It can then lead to disability and increased risks for many comorbidities and hospitalization [13]. In our study, those with sarcopenia had significantly lower scores on the EQ-VAS and the EQ-5D index score. After adjusting for other factors known to be related to HRQOL, sarcopenic men had higher rates of some or extreme problems in self-care, and sarcopenic women had higher rates of some or extreme problems in self-care, usual activities, and anxiety/depression.

Two other studies on sarcopenia in the Korean population were reported previously. Koo et al. [14] investigated the impact of sarcopenia and obesity on pulmonary function and HRQOL in male patients with chronic obstructive pulmonary disease. Both sarcopenia and obesity were independent risk factors for worsening lung function. The results showed that sarcopenia was associated with poorer QOL and obesity was related to better QOL. Another study showed the association of sarcopenic obesity with several indices of psychological health and QOL [15]. They reported that sarcopenic obesity was associated with adverse psychological health and lower QOL compared to normal population. Unlike the previous two studies, our study focused on the 
effect of sarcopenia on HRQOL in the Korean elderly population, regardless of comorbidities. We also analyzed men and women separately, because the effect of sarcopenia may be different in men and women. As a result, we confirmed that there is a significant association between sarcopenia and HRQOL in the elderly, and the impact of sarcopenia on HRQOL seemed to be greater in women than in men. One of the causes for higher impact on women's HRQOL may be a higher incidence of sarcopenic obesity in women.

In addition to sarcopenia, obesity is one of the important changes in body composition that accompanies aging processes. Normal aging is associated with a progressive increase in visceral abdominal fat mass [16]. Obesity has been shown to be linked to a higher risk for disability and comorbidity and a decreased quality of life in the elderly [17]. Obesity and sarcopenia might interact to potentiate their detrimental effects on physical disability, morbidity, and mortality $[18,19]$. In our study, women with sarcopenia had an increased waist circumference and a higher BMI than women without sarcopenia. In other words, unlike elderly men, elderly women showed a tendency for sarcopenic obesity. This difference between men and women might affect gender differences in the association between sarcopenia and HRQOL, so more research is needed to clarify the significance of sarcopenic obesity and the mechanisms that might generate gender differences.

This study has some limitations. First, the definition of sarcopenia is based only on the muscle mass. Since Baumgartner et al. [5] originally defined sarcopenia as an excessive loss of muscle mass associated with aging processes, international groups have provided revised definitions of sarcopenia including an evaluation of both muscle function and mass $[3,20]$. Our study was conducted using data from a nationwide survey that did not include an evaluation of muscle function. Second, it was not possible to analyze the effect of sarcopenia on HRQOL in the population with specific comorbidities such as cancer, chronic obstructive pulmonary disease, diabetes, and hypertension. This is the cross-sectional study that measures the proportion of a population with a particular exposure or disease at a single point in time. Therefore, the disease status of each participant is all different, and this makes it difficult to evaluate the effect of sarcopenia in homogeneous group of patients with specific diseases. Although these are limitations to the study, the results could be significant in terms of evaluating the associations between sarcopenia and HRQOL, targeted toward a representative elderly Korean population.

In conclusion, sarcopenia has become a major adverse health condition of elderly subjects and contributes to deteriorations in their physical and functional health. We found that there was a significant association between sarcopenia and impairments in HRQOL in the elderly Korean population. Therefore, efforts to treat and prevent sarcopenia might improve the quality of life for elderly people, and approaches considering gender differences in sarcopenia and obesity might be needed.

\section{KEY MESSAGE}

1. The overall prevalence of sarcopenia was $6.6 \%$ in Korean people aged 60 years or older, and sarcopenia was more common in men than in women.

2. Sarcopenia was associated with impairments in self-care for men, and with impairments in selfcare, usual activities, and anxiety/depression for women.

3. Sarcopenia has shown a more negative impact on self-care among women than among men (adjusted odds ratio 1.99 for men and 4.49 for women).

\section{Conflict of interest}

No potential conflict of interest relevant to this article was reported.

\section{Acknowledgments}

The statistical consultation was supported by a grant of the Korea Health Technology R\&D Project through the Korea Health Industry Development Institute (KHIDI), funded by the Ministry of Health and Welfare, Korea (HI14C1062).

\section{REFERENCES}

1. What quality of life? The WHOQOL group. World Health 
Organization Quality of Life Assessment. World Health Forum 1996;17:354-356.

2. Zamboni M, Zoico E, Scartezzini T, et al. Body composition changes in stable-weight elderly subjects: the effect of sex. Aging Clin Exp Res 2003;15:321-327.

3. Cruz-Jentoft AJ, Baeyens JP, Bauer JM, et al. Sarcopenia: European consensus on definition and diagnosis: report of the European Working Group on sarcopenia in older people. Age Ageing 2010;39:412-423.

4. Morley JE, Anker SD, von Haehling S. Prevalence, incidence, and clinical impact of sarcopenia: facts, numbers, and epidemiology-update 2014. J Cachexia Sarcopenia Muscle 2014;5:253-259.

5. Baumgartner RN, Koehler KM, Gallagher D, et al. Epidemiology of sarcopenia among the elderly in New Mexico. Am J Epidemiol 1998;147:755-763.

6. Beaudart C, Rizzoli R, Bruyere O, Reginster JY, Biver E. Sarcopenia: burden and challenges for public health. Arch Public Health 2014;72:45.

7. Guralnik JM, Ferrucci L, Pieper CF, et al. Lower extremity function and subsequent disability: consistency across studies, predictive models, and value of gait speed alone compared with the short physical performance battery. J Gerontol A Biol Sci Med Sci 2000;55:M221-M231.

8. Korean Centers for Disease Control and Prevention. Korean National Health and Nutrition Examination Survey [Internet]. Cheongju (KR): Korean Centers for Disease Control and Prevention, c2015 [cited 2017 Sep 25]. Available from: http://knhanes.cdc.go.kr/knanes/index.do.

9. Rabin R, de Charro F. EQ-5D: a measure of health status from the EuroQol Group. Ann Med 2001;33:337-343.

10. Kim MH, Cho YS, Uhm WS, Kim S, Bae SC. Cross-cultural adaptation and validation of the Korean version of the EQ-5D in patients with rheumatic diseases. Qual Life Res 2005;14:1401-1406.

11. Kim TN, Yang SJ, Yoo HJ, et al. Prevalence of sarcopenia and sarcopenic obesity in Korean adults: the Korean sarcopenic obesity study. Int J Obes (Lond) 2009;33:885-892.

12. Kim YS, Lee Y, Chung YS, et al. Prevalence of sarcopenia and sarcopenic obesity in the Korean population based on the Fourth Korean National Health and Nutritional Examination Surveys. J Gerontol A Biol Sci Med Sci 2012;67:1107-1113.

13. Brown JC, Harhay MO, Harhay MN. Sarcopenia and mortality among a population-based sample of community-dwelling older adults. J Cachexia Sarcopenia Muscle 2016;7:290-298.

14. Koo HK, Park JH, Park HK, Jung H, Lee SS. Conflicting role of sarcopenia and obesity in male patients with chronic obstructive pulmonary disease: Korean National Health and Nutrition Examination Survey. PLoS One 2014;9:e110448.

15. Cho Y, Shin SY, Shin MJ. Sarcopenic obesity is associated with lower indicators of psychological health and quality of life in Koreans. Nutr Res 2015;35:384-392.

16. Adams KF, Schatzkin A, Harris TB, et al. Overweight, obesity, and mortality in a large prospective cohort of persons 50 to 71 years old. N Engl J Med 2006;355:763-778.

17. Osher E, Stern N. Obesity in elderly subjects: in sheep's clothing perhaps, but still a wolf! Diabetes Care 2009;32 Suppl 2:S398-S402.

18. Zamboni M, Mazzali G, Fantin F, Rossi A, Di Francesco V. Sarcopenic obesity: a new category of obesity in the elderly. Nutr Metab Cardiovasc Dis 2008;18:388-395.

19. Choi KM. Sarcopenia and sarcopenic obesity. Korean J Intern Med 2016;31:1054-1060.

2o. Fielding RA, Vellas B, Evans WJ, et al. Sarcopenia: an undiagnosed condition in older adults. Current consensus definition: prevalence, etiology, and consequences. International working group on sarcopenia. J Am Med Dir Assoc 2011;12:249-256. 